\title{
Notas sobre la sustentabilidad y la responsabilidad social empresarial del turismo de naturaleza en la costa de Jalisco
}

\author{
Rosa María Chávez Dagostino \\ Rodrigo Espinoza Sánchez \\ Edmundo Andrade Romo \\ Centro Universitario de la Costa Universidad de Guadalajara
}

Tatiana Gómez Morales*

Universidad Tecnológica

de Bahía de Banderas

\section{Resumen}

Aunque existen varias definiciones de turismo de naturaleza, la mayoría concuerda en que es una forma de turismo que promueve una relación más estrecha con la naturaleza, y un interés por la conservación de los recursos naturales y culturales del área donde se desarrolla. Se considera un segmento de rápido crecimiento y supone un modo más responsable de turismo. En este mercado competitivo, las empresas tienen mayor responsabilidad, en tanto que los turistas son cada vez más sensibles en cuestiones medioambientales y sociales. Con el fin de evaluar el papel del turismo de naturaleza en la costa de Jalisco, relacionado con los supuestos de responsabilidad ambiental, social y económica, se analizó la responsabilidad social empresarial (RSE) de cinco pequeñas y medianas empresas de turismo de naturaleza situadas en los municipios costeros. Se empleó el software ADVALUA, una herramienta de la economía social utilizada en la Unión Europea que permite, entre otras cosas, evaluar la sustentabilidad. Se concluyó la carencia general de buenas prácticas de rse en los tres ámbitos: social, ambiental y económico, condición que compromete la sustentabilidad del turismo de naturaleza, no obstante, estuvieron mejor representadas las acciones sociales.

\section{PALABRAs Clave}

Empresa, impacto, responsabilidad social, sustentabilidad. 


\title{
Notes on the entrepreneurial tourism on the coast of Jalisco, its sustainability and social responsibility
}

\author{
Rosa María Chávez Dagostino \\ Rodrigo Espinoza Sánchez \\ Edmundo Andrade Romo \\ Centro Universitario de la Costa Universidad de Guadalajara
}

Tatiana Gómez Morales

Universidad Tecnológica

de Bahía de Banderas

\begin{abstract}
There are various definitions of eco-tourism but there is one that pervades and is the one that sees it as a form of tourism that promotes a closer contact with nature and encourages the preservation of natural and cultural resources. Eco-tourism is growing rapidly and is seen as more sensitive to environmental and social issues than other kinds of tourism. Companies in this competitive market have more demands to fulfill the expectation on nature preservation. To be able to evaluate the role of eco-tourism on the coast of Jalisco and its environmental, social and economic responsibilities, we analyzed CRS in five small and medium tourist enterprises by the sea. We use a tool of social economy used in the European Union, ADVALUA software, which allows us to assess sustainability, among other things. We concluded that there is a lack of good practices of corporate social responsibility in three areas: social, environmental and economic issues that compromise the sustainability of eco-tourism in the area, but some good social actions were detected.
\end{abstract}

\section{KEY WORDS}

Corporate, impact, social responsibility, sustainability. 


\section{Introducción}

En la región costera del estado de Jalisco, México, constituida por cinco municipios y distinguida por una alta migración, ruralidad y desarrollo turístico polarizado en el municipio de Puerto Vallarta, Chávez-Dagostino et al. (2011) identificaron y caracterizaron en la zona 59 pequeñas y medianas empresas (pyme), privadas y comunitarias, dedicadas al turismo de naturaleza, algunas desarrolladas con apoyos del gobierno federal. De las 30 actividades de turismo de naturaleza encontradas, dominan las tipificadas como de aventura, seguidas por las actividades de ecoturismo, mientras que el turismo rural está escasamente representado.

Si bien en los últimos años ha sido otorgada una gran cantidad de apoyos técnicos y financieros por parte de entidades de gobierno de distintos niveles y organismos no gubernamentales, se carece de un registro que identifique a las empresas turísticas de naturaleza, su localización y sus características. Sin embargo, estas últimas, como el tamaño y temas de planeación, operación, capacitación e infraestructura, relacionadas con un bajo impacto ambiental y su contribución a la conservación de los recursos naturales y culturales, se dan por hecho y en general no se verifican, en el supuesto de que el turismo de naturaleza es una forma más responsable de hacer turismo.

Como continuación del trabajo de identificación y caracterización de las empresas de turismo de naturaleza en la costa de Jalisco durante 2010 y 2011, se planteó evaluar su desempeño ambiental, social y económico, ámbitos que representan los pilares del desarrollo sustentable y constituyen indicadores clave de responsabilidad social de las organizaciones.

El objetivo de este trabajo es hacer un estudio exploratorio con el fin de evaluar el papel del turismo de naturaleza en la costa de Jalisco, considerando los supuestos de responsabilidad ambiental, social y económica de las pyme y utilizando una herramienta interactiva y sencilla para evaluar la responsabilidad social empresarial (RSE). Este análisis permitirá a cada empresa valorar los comportamientos responsables que desarrolla actualmente, así como los faltantes; además de reorientar sus acciones como elemento indispensable de la competitividad en el turismo y a la vez valorar las políticas relacionadas con el desarrollo del turismo de naturaleza 62 en la región. 
En los siguientes apartados se hace una revisión de la literatura centrada en la noción de turismo de naturaleza y RSE, y en las diversas formas de desempeño empresarial en materia de RSE en empresas turísticas; se describe asimismo el turismo de naturaleza como un ámbito de concreción de los principios de la RSE en el sector turístico, sin profundizar en el debate de estos temas, ya que no es el propósito de este artículo. Luego se expone la metodología adoptada en el trabajo, los resultados, la discusión y las conclusiones.

\section{Revisión de la literatura \\ El turismo de naturaleza}

La antigua concepción errónea acerca de que el turismo era una industria ambientalmente benigna y llamada con frecuencia "sin chimeneas" llevó a una pobre y lenta integración de elementos de responsabilidad ambiental y social en su planeación y desarrollo. Hasta fines de los años ochenta el sector empezó a tener conciencia del asunto, de la importancia del turismo sostenible y de la nueva dirección que el turismo tomaría. La creencia paralela de que el turismo alternativo era la respuesta para atender los impactos negativos del sector perdió fuerza en la década de los noventa: se admitió que había una distancia significativa entre lo esperado y lo obtenido. Una nueva forma de pensamiento surgió: el turismo sustentable requería un esfuerzo colectivo y consciente por parte de todos los actores del sector, incluso de los diseñadores de políticas gubernamentales y planificadores, con la intención de priorizar los temas ambientales y sociales en sus acciones cotidianas. En los años siguientes se reconoció que el sector turístico, en particular los hoteles como empresas clave, necesitaba hacerse cargo de las obligaciones ambientales y sociales (Kasim, 2006), sin embargo, aunque el turismo de naturaleza había sido sugerido como una opción más responsable —en sentido amplio— de hacer turismo, pocos estudios se han efectuado para demostrarlo.

A pesar de que existen numerosas definiciones del turismo de naturaleza, la mayoría coincide en que es un tipo de turismo con una fuerte dependencia de la naturaleza y preocupado por la conservación de los recursos naturales y culturales en el área donde se lleva a cabo. La Secretaría de Turismo en México (Sectur, 2007) lo define como aquellos viajes designados para desarrollar actividades recreativas en contacto directo con las expresiones naturales y culturales, con una actitud 
positiva para participar en su conservación; ha sido fuertemente promocionado en una gran variedad de áreas rurales del país, con el propósito de reforzar pequeñas y medianas organizaciones locales, así como por su responsabilidad social y ambiental. Uno de sus principales logros tiene que ver con los temas ambientales, sobre todo con su papel en la promoción del cambio de racionalidad entre los habitantes de las localidades respecto a la importancia de preservar los ecosistemas.

\section{La responsabilidad social de las empresas}

Críticos y proponentes del concepto de RSE todavía debaten una variedad de temas relacionados, que van desde las ideas de Milton Friedman sobre libertad, en los setenta, hasta conceptos neoliberales que incluyen el propósito fundamental, la naturaleza de los negocios y los motivos cuestionables por los que las empresas se adhieren a ello (Nocera, 2006). Aunque la ideología que dio origen al término RSE data de principios del siglo pasado, a partir de los años noventa ha cobrado fuerza y evolucionado constantemente tras el advenimiento de la globalización, la aceleración de la actividad económica, la conciencia ecológica y el desarrollo de nuevas tecnologías. En general, el mundo de los negocios ha cambiado su conceptualización en cuanto a los objetivos que deben perseguirse: de una misión exclusivamente económica a una socioeconómica con una seria responsabilidad moral con la sociedad.

De la Torre (2005) define la RSE como una forma de entregar bienes y servicios lícitos a la sociedad; de proporcionar el valor agregado que espera cada participante del negocio, y de desarrollar personas mediante la relación de la inversión y el trabajo. Esto a su vez exige del inversionista rendimiento justo, creatividad e innovación, y estabilidad y permanencia, requerimientos que se vinculan directamente con vida digna, desarrollo integral y seguridad y retiro para el trabajador, todos conciliados en un solo eje de competitividad y confianza. Señala también que en Latinoamérica, a diferencia del resto del mundo, existe una tendencia basada en demandas sociales a través de la unificación normativa, el abatimiento de la pobreza y un buen gobierno.

La RSE se precisa asimismo como la contribución activa y voluntaria de las organizaciones en la mejora ambiental, social y económica, que promueve una posición competitiva y valor de mercado, incluyendo los impactos positivos que estas 
acciones generan en las diferentes áreas con las que la compañía se relaciona (Alea-García, 2007). Se pueden encontrar múltiples definiciones en artículos académicos, reportes de empresas y documentos políticos, lo que vuelve confuso el tema, pero al mismo tiempo demuestra la debilidad del concepto (Argandoña y Weltzien, 2009). Tradicionalmente se distinguen tres tipos de responsabilidad: económica, social y ambiental (Carroll, 1979), que a su vez se engloban en el término de responsabilidad social y son una distinción práctica que ayuda a organizar la información; sin embargo, no refleja todo lo que conlleva la responsabilidad social.

\section{El desempeño de las empresas}

Un futuro sostenible significa mercados que premien el desempeño a largo plazo. Entraña una visión responsable de las prácticas empresariales como guía para la calidad de la firma y su gestión, lo cual implicaría que las políticas públicas y la acción ciudadana ayuden a las empresas a hacer lo correcto. La empresa como célula del modelo económico capitalista ha implementado diversas maneras de evaluar su desempeño en aras de monitorear indicadores de su gestión relacionados con la competitividad. La palabra clave para la competitividad basada en el actual conocimiento de la economía es la innovación, y las mejores compañías se han dado cuenta de que ésta y la RSE están íntimamente ligadas. Mayor atención a los problemas sociales y ambientales y mayor cooperación con otras partes interesadas son los catalizadores para desarrollar nuevos productos y servicios. La RSE no trata sólo de proteger el valor de una empresa evitando acciones que puedan poner en peligro su reputación y la lealtad de sus clientes, sino también, y cada vez más, de crear nuevos valores mediante la innovación generada por dar mayor importancia a los problemas sociales y cultivar relaciones más profundas con las partes interesadas (Verheugen, 2007).

Históricamente, la RSE ha sido abordada con mayor frecuencia por el sector minero y el de hidrocarburos, aunque en la actualidad otros sectores han incorporado en sus proyectos indicadores que manifiestan un grado variable de armonía con las poblaciones y organizaciones sociales de una región.

La RSE se aplica a diversas cuestiones, como políticas de contratación local, estrategias de comunicación y consulta, desarrollo local, negociaciones, planes de 
relaciones comunitarias, entre otras, y existen varias maneras de acreditarla con base en acuerdos internacionales sobre las condiciones laborales, que incluyen temas como la justicia social y los derechos de los trabajadores.

Algunos organismos de carácter internacional se encargan de delimitar en lo posible el concepto teórico de la RSE, y con sus principios orientan a las empresas que deciden incursionar en este campo en el marco de la sustentabilidad; entre los más destacados se encuentran Global Compact (ONU, 2007) y Global Reporting Initiative (GRI, 2010). Además, hay otras iniciativas nacionales que denotan una incursión en el tema de la RSE y que contribuyen de forma determinante a la creación y difusión de una cultura responsable entre las organizaciones empresariales en cada país: por ejemplo, como documento decisivo acerca de la RSE en Europa, sobresale el llamado "Libro Verde" (Comisión Europea, 2001); o algunas leyes económicas, como la francesa y la brasileña, desde inicios de siglo establecen como obligación jurídica de las empresas informar acerca de sus acciones de índole social. El reconocimiento de la RSE dentro de la evaluación internacional de estándares de calidad ha sido formalizado mediante la certificación SA 8000 y el WRAP, entre otros (Demirdjian et al, 2010).

La presentación de informes sobre el desempeño en materia de sustentabilidad es hoy una necesidad y, para las organizaciones, una forma importante de gestionar su impacto. Es ampliamente aceptado que éstas no sólo tienen la responsabilidad, sino también la gran capacidad de ejercer un cambio positivo en las condiciones sociales, económicas y ambientales en todo el orbe.

De acuerdo con las iniciativas y normas variadas alrededor del mundo, existen diversos métodos para medir la RSE (Correa, Flynn y Amit, 2004). Uno de los más recientes fue desarrollado entre 2004 y 2007 por la Confederación de Cooperativas de Cataluña: el programa RSE.COOP, un proyecto piloto diseñado para adaptar la economía cooperativa al cambio económico estructural. Esto involucró la revaluación de la responsabilidad social en España, utilizando un proceso que creó la marca ADVALUA para la Confederación Empresarial Española de la Economía Social (Cepes) a fin de identificar en principio aquellas compañías que aplican políticas de responsabilidad social, y elaborar memorias de sostenibilidad que incluyan los principios de GRI (2010).

En México se han realizado numerosos esfuerzos en el área, prueba de ello es la Norma Mexicana de Responsabilidad Social, de aplicación voluntaria y dirigida 
a toda organización pública o privada que desee implementar buenas prácticas de responsabilidad social, que proporciona directrices basadas en un modelo de sistema de gestión que facilita su planificación, operación, seguimiento y mejora continua (NMX-SAST-004-IMNC-2004, $1^{\circ}$ de marzo de 2005). Una cuestión fundamental de esta norma es el proceso para identificar los aspectos e impactos sociales respecto a sus partes interesadas, y orientar acerca de acciones concretas que la organización puede llevar a cabo con cada una de ellas. La norma considera relevante la evaluación y asignación de prioridades de los aspectos e impactos identificados con el propósito de establecer los objetivos de responsabilidad social desde su planificación. Asimismo, ha constituido un fundamento para otras, como la norma internacional ISO 26000, y, hasta este momento, ha mantenido su consistencia en materias primordiales (Demirdjian et al., 2010).

No obstante los logros alcanzados en este campo en México, se sigue pensando que el principal valor es uno: la obtención de beneficios económicos, situación que se acentúa en las pyme. Por lo tanto, uno de los problemas que enfrenta México es que la RSE se considera una actividad periférica por parte de las empresas y con frecuencia se confunde con filantropía.

\section{La responsabilidad social de las empresas turísticas}

El tema de la RSE está ganando impulso en todo el mundo a medida que las empresas empiezan a darse cuenta de que las partes involucradas exigen rendición de cuentas, lo que va más allá de los intereses de los accionistas. Así, las iniciativas estratégicas centradas en la mejora de su responsabilidad social y ambiental van también en aumento, no obstante, aún son incipientes y hay muy pocas prácticas de RSE en el caso del turismo, sobre todo en los hoteles. No se encontraron estudios que hayan analizado el turismo de naturaleza en relación con la RSE. Sin embargo, temas como el cambio climático y el turismo responsable han empezado a influir en la demanda de los consumidores desde hace un par de años; el hallazgo de diversos estudios realizados al respecto está variando debido a esta influencia de los consumidores.

Dado que Canadá es una importante fuente de turistas para México, las islas del Caribe, Cuba y República Dominicana, Dodds y Kuehnel (2010) hicieron un estudio 
exploratorio para evaluar el nivel de conciencia de la responsabilidad social de las actividades de los operadores de turismo masivo en el mercado canadiense, y observaron una pobre implementación de prácticas de RSE, pero dejaron pendiente el análisis de las diferencias relacionadas con el tamaño de las empresas.

\section{Metodología}

Se seleccionaron cinco empresas de turismo de naturaleza de las 59 identificadas en un estudio previo (Chávez-Dagostino et al., 2011), una por cada municipio costero, con entre cinco y diez años de operación y que manifestaran interés en participar. Las empresas elegidas representan $18 \%$ del total que cumple el requisito de tiempo de operación. Se incluyeron y se visitaron dos cooperativas - una de ellas ejido- y tres empresas privadas. El grupo de trabajo realizó algunas de las actividades que ofertan y entrevistó a los responsables, administradores, dueños 0 gerentes generales, quienes respondieron 14 preguntas elaboradas de acuerdo con indicadores propuestos en la evaluación básica del programa RSE.COOP (2007) (cuadro 1). En algunos casos fue necesario entrevistar a más de una persona en la empresa para completar la información.

En el ámbito económico, se consideraron los indicadores relevantes: si la organización dispone de una declaración formal de sus valores, misión, visión y objetivos a largo plazo, así como de un sistema de control; si prioriza las compras de recursos locales y tiene una política definida respecto a ellas; si, en términos operativos, dispone de un sistema de gestión de la calidad que además incorpore las actuaciones de la organización en RSE, y si tiene herramientas para la planificación y el seguimiento presupuestarios; si cuenta con un plan de mercadotecnia, controla sus productos/servicios, y enfoca parte de sus esfuerzos a la generación de productos/servicios y procesos innovadores, todo ello incorporando un seguimiento documentado de los resultados generados; si lleva un control de ventas, del destino de excedentes, de la distribución de deudas entre las empresas proveedoras de capital, de los impuestos y subsidios recibidos, y de la evolución de las ganancias retenidas. En el área ambiental los indicadores base son: si la empresa evidencia un compromiso explícito con el medio ambiente y establece indicadores de medida que le permitan analizar resultados y fijarse objetivos de mejora o de minimización de los impactos ambientales; si aplica mecanismos 
que favorezcan tanto una buena gestión de sus residuos sólidos, el agua y la energía, como la reducción de diferentes tipos de contaminantes (atmosféricos, lumínicos, acústicos y del suelo); si fomenta la participación, información y formación de las personas en los procesos de mejora ambiental de la actividad. En el terreno social los indicadores son: si la organización ha asumido que la transparencia para con sus grupos de interés (turistas, empleados, población local) es clave en su gestión diaria y establece canales de comunicación y diálogo con ellos para conocer sus necesidades y darles solución; si ha definido valores corporativos y principios de actuación éticos para cada grupo de interés que guíen su actividad a corto y largo plazos; si cuenta con mecanismos que garanticen la igualdad en la selección, contratación, formación, promoción y retribución de los miembros de la organización; si hay un esfuerzo planeado para colaborar en el desarrollo de la comunidad local mediante su participación activa en el tejido asociativo local, acciones de patrocinio y acción social, o acuerdos de colaboración con otras organizaciones o empresas; si asume en su relación con los clientes aspectos que trascienden los puramente legales u obligatorios (inversión en innovación, certificaciones, servicio de atención, etc.) (cuadro 1).

Con las respuestas se elaboró una base de datos con el software ADVALUA (evaluación básica), que proporcionó resultados en las dimensiones social, económica y ambiental. El grupo de trabajo asignó un puntaje para cada una de las 14 respuestas en los tres ámbitos, con valores de 0 a 3 (establecido por el programa), donde 0 implica ausencia absoluta y 3 presencia. Los valores intermedios expresan cierto grado de logro: valores menores a 1 reflejan una baja responsabilidad social de la empresa, de 1 a 2 implican un desempeño medio, y arriba de 2 equivalen a un buen desempeño. Con estos valores se calculó el promedio total por empresa y por ámbito (económico, ambiental y social). Se empleó una técnica estadística no métrica: Multidimensional Scaling (MDS), a fin de relacionar los puntajes promedio por área y empresa representados como distancias interelementales y establecer su similitud (Fenton y Pearce, 1988) con el programa PAST 2.09 (Hammer, Harper y Ryan, 2001), y se obtuvo el valor de estrés (Kruskal, 1964). 
CUADRO 1. Indicadores del cuestionario general de evaluación básica de RSE, software ADVALUA (Cepes, Proyecto Equal)

\begin{tabular}{lccl}
\hline Económico & Misión, Visión, Valores y objetivos estratégicos & $\square 0 \square 1 \square 2 \square 3$ \\
Económico & Uso de recursos locales & $\square 0 \square 1 \square 2 \square 3$ \\
Económico & Sistemas de gestión & $\square 0 \square 1 \square 2 \square 3$ \\
Económico & Marketing e innivación & $\square 0 \square 1 \square 2 \square 3$ \\
Económico & Control económico & $\square 0 \square 1 \square 2 \square 3$ \\
Ambiental & Política y objetivos ambientales & $\square 0 \square 1 \square 2 \square 3$ \\
Ambiental & Gestión de residuos agua y energía & $\square 0 \square 1 \square 2 \square 3$ \\
Ambiental & Reducción diferentes contaminantes & $\square 0 \square 1 \square 2 \square 3$ \\
Ambiental & Información y formación ambiental & $\square 0 \square 1 \square 2 \square 3$ \\
Social & Diálogo y transparencia grupos de interés & $\square 0 \square 1 \square 2 \square 3$ \\
Social & Valores corporativos y principios éticos & $\square 0 \square 1 \square 2 \square 3$ \\
Social & Igualdad de oportunidades & $\square 0 \square 1 \square 2 \square 3$ \\
Social & Comunidad local & $\square 0 \square 1 \square 2 \square 3$ \\
Social & Satisfacción de la clientela & $\square 0 \square 1 \square 2 \square 3$ \\
\hline
\end{tabular}

Fuente: RSE.COOP, 2007.

\section{Resultados}

Las características y los puntajes de las cinco empresas evaluadas fueron los siguientes:

a) Ecotours de México es una empresa privada con 17 empleados localizada en el municipio de Puerto Vallarta, creada en 1996. Obtuvo 32 de 42 puntos posibles en la evaluación de RSE y su mejor desempeño fue en el área ambiental, seguido por la económica. Oferta actividades de observación de mamíferos marinos, actividades en campamentos tortugueros, aventuras con cocodrilos y buceo; todas guiadas por grupos de biólogos y naturalistas (figura 1 , cuadro 2 ).

b) El Cielito, en el municipio de Cabo Corrientes, es una cooperativa que se autodenomina ecológica, con 34 socios, que forma parte de un ejido. Inició sus servicios en 2006 y ofrece hospedaje y restaurante frente al mar, que es parte del santuario tortuguero más grande del país; caminata en el pueblo y por la playa, participación en actividades del campamento tortuguero y esnórquel, aunque la mayoría de las actividades se diseña a petición del 


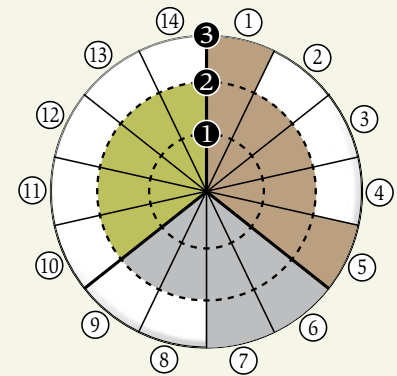

a) Ecotours de México

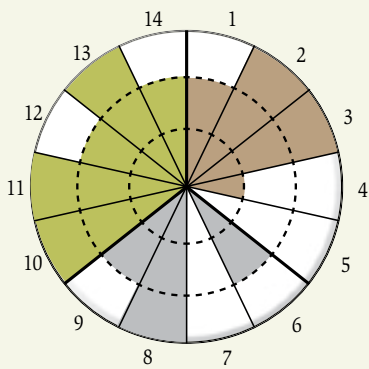

d) Ecoadventures

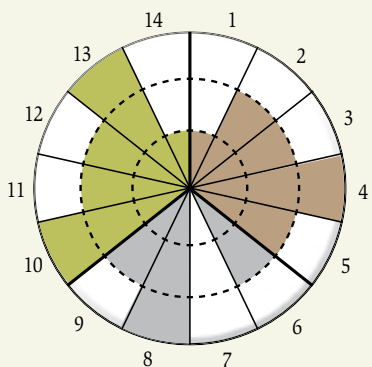

c) El Rancho Andrea

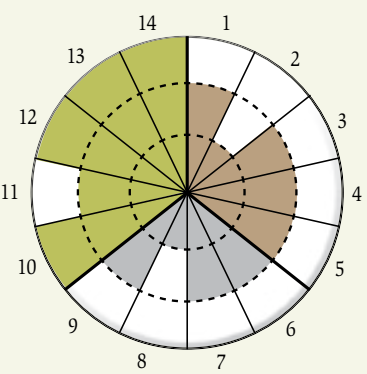

b) El Cielito

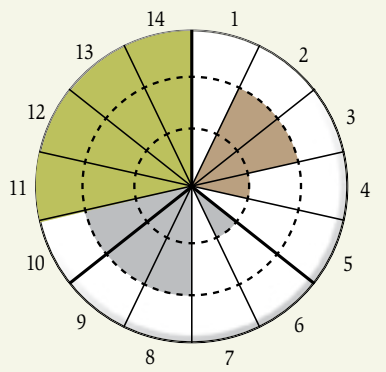

e) La Sociedad Cooperativa de Servicios Turísticos Miguel López de Legazpi

FIGURA 1. Puntaje de RSE obtenido por empresa en los ámbitos económico (1-5), ambiental (6-9) y social (10-14)

turista. Su puntaje fue de 30 (desempeño medio); su mejor resultado lo obtuvo en el ámbito social, mientras que el más bajo, en el ambiental.

c) El Rancho Andrea es una empresa privada que abrió en 2000 en el municipio de Tomatlán y opera con cinco empleados. Oferta kayaking, natación, esquí acuático, pesca y canotaje en la presa Cajón de Peñas, y actividades terrestres como paseos a caballo y observación de aves. Obtuvo 28 puntos (nivel medio) y su mejor desempeño fue en el terreno social. 
d) Ecoadventures es una empresa privada que organiza viajes grupales para acampar en el Rancho Cadillac, cerca de Barra de Navidad. Ofrece rappel, paseos en bote, observación de flora y fauna, buceo y esnórquel, además de caminatas por el río y el manglar. Comenzó sus actividades en 2000 y labora solamente con una persona la mayor parte del año. Logró 34 puntos, el mayor puntaje total de todas las empresas evaluadas; su desempeño más bajo lo tuvo en el campo económico.

e) La Sociedad Cooperativa de Servicios Turísticos (SCST) Miguel López de Legazpi es una cooperativa con 29 socios que inició sus operaciones en 2000. Se dedica principalmente a la renta de botes, a la observación de vida marina y aves, y a la pesca en la bahía de Navidad. Su puntaje resultó el menor de todos: 28 puntos (que es muy cercano al desempeño bajo). Las áreas donde obtuvo las calificaciones más bajas fueron la económica y la ambiental.

CUADRo 2. Puntaje promedio obtenido por área y por empresa

\begin{tabular}{|c|c|c|c|c|c|c|}
\hline $\begin{array}{c}\text { Empresa } \\
\text { Área }\end{array}$ & $\mathrm{a}$ & $\mathrm{b}$ & $\mathrm{c}$ & d & e & Promedio \\
\hline Económica & 2.4 & 1.8 & 2.0 & 1.8 & 0.8 & 1.76 \\
\hline Ambiental & 2.5 & 1.75 & 1.75 & 2.75 & 1.25 & 2.0 \\
\hline Social & 2.0 & 2.8 & 2.2 & 2.6 & 2.8 & 2.48 \\
\hline Promedio & 2.3 & 2.11 & 1.9 & 2.38 & 1.6 & 2.08 \\
\hline
\end{tabular}

\section{Discusión}

El área social (preguntas 10-14) en general logró el mayor puntaje total en comparación con las otras dos (cuadros 1 y 2, figura 1). Las preguntas con los puntajes más bajos fueron la 5 en el ámbito económico y la 7 en el ambiental, lo que puede explicarse como resultado de la falta de experiencia y entrenamiento, y da la impresión de que estas acciones son irrelevantes para tres de las empresas analizadas, que obtuvieron los valores más altos de su evaluación en el ámbito social, sobre todo en las preguntas 10 y 13. En este campo, Ecotours de México tuvo una pobre puntuación 
debido a que se localiza en Puerto Vallarta, el municipio más urbanizado de la costa, en tanto que los otros turoperadores se ubican en pueblos pequeños donde hay mayor interacción con la comunidad local. El desempeño más bajo en general fue en el ámbito económico. Esrock y Leichty (1998) encontraron correlación entre el tamaño de una compañía y la extensión en la que promueve la responsabilidad social. Este hallazgo no puede aclarar por qué Ecoadventures consiguió la mejor puntuación con un solo trabajador, ni por qué la SCST Miguel López de Legazpi (la segunda en tamaño por el número de empleados) obtuvo el puntaje total más bajo. Las cooperativas exhibieron un alto desempeño en el ámbito social. El proceso de MDS reveló una relación significativa, mediante la matriz de distancias, entre las empresas $a$ - $d$ (grupo 1 , privadas) y $b$-c (grupo 2, cooperativas). Las empresas privadas ( $a$ - $d$ ) mostraron el mejor desempeño general en RSE, hecho que puede ser explicado principalmente por su desempeño económico y ambiental. Orlitzky, Schmidt y Rynes (2003) realizaron un análisis sobre el desempeño corporativo social y económico. Asumieron que la mayoría de las teorías sobre la relación entre el desempeño corporativo de la responsabilidad social/ambiental y económica están fracturadas y son muy variables, por lo que no pueden hacerse conclusiones generalizadas. Por último, determinaron que existe mayor certeza respecto a la relación desempeño ambiental-social y financiero, y una relación positiva significativa entre las variables sociales-ambientales y financieras. Sin embargo, también encontraron que, en las 52 empresas que evaluaron, "lo social" tiene más valor frente a "lo ambiental" en este tema, situación comparable con lo observado en este estudio.

Sobre el programa ADVALUA, debe decirse que es una herramienta sencilla que produce resultados rápidos con pocas preguntas, no obstante puede resultar complejo aplicar el cuestionario en las comunidades rurales, difícil de responder para algunas empresas o de calificar para el grupo aplicador. Esto tiene que ver con el tamaño de la empresa y si está localizada en áreas rurales o urbanas. Por ejemplo, acerca del indicador ambiental "promover buenas prácticas relacionadas con el manejo de la basura, agua y electricidad”, una respuesta negativa obtendría un valor de 0 , pero en una empresa rural puede significar la no disponibilidad de los servicios o un consumo muy bajo de productos procesados (basura). Las empresas en estas condiciones tendrían un puntaje bajo que no reflejaría la realidad. Por ello se sugiere ampliar o modificar los indicadores en caso necesario, y, al grupo evaluador, asignar puntaje de acuerdo con el "hecho comprobado" para cada empresa. 


\section{Conclusiones}

- La RSE de las empresas de turismo de naturaleza evaluadas en la costa de Jalisco está mejor representada por las acciones sociales, seguidas de las ambientales y las económicas

- Un resultado no esperado fue que las organizaciones con formato de cooperativa demostraron niveles más bajos de desempeño comparadas con las empresas privadas

- En general hay escasez de buenas prácticas de responsabilidad social, sobre todo en el área económica

- El turismo de naturaleza crea pocos empleos en la zona, una gran proporción es inestable, y no existe la equidad de género en las empresas de turismo de naturaleza evaluadas, ya que el género masculino es dominante en el segmento en esta región

- El área ambiental obtuvo un puntaje general de desempeño medio, lo cual puede contradecir la actuación esperada del turismo de naturaleza cuando trata de disminuir los impactos ambientales negativos

- Desde el enfoque de la RSE, la sustentabilidad del turismo de naturaleza en la región estudiada es cuestionable en general, lo que probablemente esté relacionado con el tamaño de las empresas.

\section{AgRADECIMIENTOS}

Agradecemos al doctor Fabio Cupul Magaña, investigador del Centro Universitario de la Costa, por su asesoría en el corrimiento de la prueba estadística de escalamiento multidimensional. 


\section{FUENTES CONSULTADAS}

Alea-García, A. (2007). "Responsabilidad social empresarial. Su contribución al desarrollo sostenible”. Revista Futuros, V (17) [en línea]. Disponible en: http://www. revistafuturos.info/raw_text/raw_futuro17/resp_social_empr.pdf [2011, 2 de febrero].

Argandoña, A. y H. Weltzien (2009). "Corporate Social Responsibility: One Size does not Fit All. Collecting Evidence from Europe”. Journal of Business Ethics, 89, 221-234.

Carroll, A. B. (1979). "A Three-Dimensional Conceptual Model of Corporate Performance". Academy of Management Review, 4 (4), 497-505.

Chávez-Dagostino, R. M., et al. (2011). Impacto de las empresas de turismo de naturaleza en la costa de Jalisco. Informe técnico final. Guadalajara: Consejo Estatal de Ciencia y Tecnología de Jalisco/Universidad de Guadalajara.

Comisión Europea (2001). Libro Verde. La normativa de gobierno corporativo de la ue [en línea]. Bruselas. Disponible en: http://ec.europa.eu/internal_market/ company/docs/modern/com2011-164_es.pdf [2011, 18 de mayo].

Correa, M. E., S. Flynn y A. Amit (2004). Responsabilidad social corporativa en América Latina: una visión empresarial. Santiago de Chile: División de Desarrollo Sostenible y Asentamientos Humanos-Comisión Económica para América Latina y el Caribe (Serie Medio Ambiente y Desarrollo 85).

Demirdjian, M. T., et al. (2010). "Implicaciones socioeconómicas de la responsabilidad social empresarial en México, casos: Televisa y TV Azteca”. Observatorio de la Economía Latinoamericana, 131, 20 de mayo de 2010 [en línea]. Disponible en: http://www.eumed.net/cursecon/ecolat/mx/2010/plsu.htm [2011, 15 de junio].

Dodds, R. y J. Kuehnel (2010). "CSR among Canadian mass Tour Operators: Good Awareness but Little Action”. International Journal of Contemporary Hospitality Management, 22 (2), 221-244.

Esrock, S. L. y G. B. Leichty (1998). "Social Responsibility and Corporate Web Pages: Self Presentation or Agenda Setting”. Public Relation Review, 24 (3), 305-319.

Fenton, M. y P. Pearce (1988). "Multidimensional Scaling and Tourism Research". Annals of Tourism Research, 15 (2), 236-254. 
Notas sobre la sustentabilidad y la responsabilidad social empresarial del turismo de naturaleza en la costa de Jalisco

Frankental, P. (2001). “Corporate Social Responsibility - a PR Invention?” Corporate Communications, 6 (1), 18-23.

Friedman, M. (1970). "The Social Responsibility of Business is to Increase its Profits". The New York Times Magazine, 13 de septiembre de 1970 [en línea]. Disponible en: http://www.colorado.edu/studentgroups/libertarians/issues/friedmansoc-resp-business.html [2008, 3 de julio].

GRI (2010). GRI Sustainability Reporting Statistics-2010 [en línea]. Global Reporting Initiative. Disponible en: https://www.globalreporting.org/resourcelibrary/ GRI-Reporting-Stats-2010.pdf [2011, 1 de marzo].

H. Congreso de la Unión (1994). "Ley General de Sociedades Cooperativas". Diario Oficial de la Federación, 3 de agosto de 1994. México: Secetaría de Gobernación.

Hammer, Ø., D. A. T. Harper y P. D. Ryan (2001). "PAST: Paleontological Statistics Software Package for Education and Data Analysis”. Palaeontologia Electronica, 4 (1) [en línea]. Disponible en: http://palaeo-electronica.org/2001_1/past/ issue1_01.htm [2011, 7 de abril].

Kasim, A. (2006). "The Need for Business Environmental and Social Responsibility in the Tourism Industry". International Journal of Hospitality and Tourism Administration, 7 (1), 1-22.

Kruskal, J. B. (1964). "Nonmetric Multidimensional Scaling: A Numerical Method". Psychometrika, 2, 115-129.

Nocera, J. (2006). "The Paradoxes of Businesses as Do-Gooders”. The New York Times, 11 de noviembre de 2006 [en línea]. Disponible en: http://www.nytimes. com/2006/11/11/business/11 nocera.html?pagewanted = all [2011, 3 de julio].

ONU (2007). ¿Qué es el pacto mundial? [en línea]. Organización de las Naciones Unidas.

Disponible en: http://www.unglobalcompact.org/Languages/spanish/index. html [2010, 6 de octubre].

Orlitzky, M., F. L. Schmidt y S. L. Rynes (2003). "Corporate Social and Financial Performance: A Meta-Analysis”. Organization Studies, 24 (3), 403-441.

RSE.COOP (2007). Manual para el tutor del programa RSE.COOP y software ADVALUA. Responsabilitat Social de les empreses en l'economia Cooperativa. Fondo Social Europeo/Unión Europea/Equal.

Sectur (2007). Elementos metodológicos para la evaluación del impacto económico, social y ambiental del turismo de naturaleza en México. Informe ejecutivo. México: Secretaría de Turismo/Universidad Nacional Autónoma de México. 
Torre, G. A. de la (2005). "La responsabilidad social empresarial - Detonante de la productividad laboral” [en línea]. Ponencia presentada en el IV Foro Laboral Internacional, Ciudad de México, 15 de noviembre de 2005. Disponible en: http://www.coparmex.org.mx/eventos/forolaboral/4tointernacional/ programa.htm [2011, 4 de agosto].

Verheugen, G. (2007). "CSR and Competitiveness: A View from the European Commission”, en A. MacGillivray, P. Begley y S. Zadek (eds). The State of Responsible Competitiveness 2007. Making Sustainable Development Count in Global Markets. Londres: Accountability, 111-115. 